\title{
On Fibonacci Functions with Period $k$
}

\author{
Banyat Sroysang \\ Department of Mathematics and Statistics, Faculty of Science and Technology, Thammasat University, Pathumthani 12121, Thailand \\ Correspondence should be addressed to Banyat Sroysang; banyat@mathstat.sci.tu.ac.th
}

Received 6 February 2013; Accepted 4 April 2013

Academic Editor: Gabriele Bonanno

Copyright (C) 2013 Banyat Sroysang. This is an open access article distributed under the Creative Commons Attribution License, which permits unrestricted use, distribution, and reproduction in any medium, provided the original work is properly cited.

A function $f: \mathbb{R} \rightarrow \mathbb{R}$ is said to be a Fibonacci function if $f(x+2)=f(x+1)+f(x)$ for all $x \in \mathbb{R}$. In 2012, some properties on the Fibonacci functions were presented. In this paper, for any positive integer $k$, a function $f: \mathbb{R} \rightarrow \mathbb{R}$ is said to be a Fibonacci function with period $k$ if $f(x+2 k)=f(x+k)+f(x)$ for all $x \in \mathbb{R}$; we present some properties on the Fibonacci functions with period $k$.

\section{Introduction}

Presently, there are many research articles about Fibonacci numbers (see [1]). Fibonacci numbers are also involved in the golden ratio (see [2]). In 2008, Kim and Neggers [3] studied Fibonacci means. In 2009, Jung [4] studied Hyers-Ulam stability of Fibonacci functional equation. In 2010, Han et al. [5] studied a Fibonacci norm of positive integers. In 2012, Han et al. [6] studied Fibonacci sequences in groupoids. Moreover, they [7] gave some properties on Fibonacci functions; a function $f: \mathbb{R} \rightarrow \mathbb{R}$ is said to be a Fibonacci function if $f(x+2)=f(x+1)+f(x)$, for all $x \in \mathbb{R}$, using the concept of $f$-even and $f$-odd functions. They also showed that if $f$ is a Fibonacci function, then $\lim _{x \rightarrow \infty} f(x+1) / f(x)=(1+\sqrt{5}) / 2$.

In this paper, for any positive integer $k$, a function $f$ : $\mathbb{R} \rightarrow \mathbb{R}$ is said to be a Fibonacci function with period $k$ if $f(x+2 k)=f(x+k)+f(x)$ for all $x \in \mathbb{R}$; we present some properties on the Fibonacci functions with period $k$ using the concept of $f$-even and $f$-odd functions with period $k$. Moreover, we also present some properties on the odd Fibonacci functions with period $k$.

\section{Fibonacci Functions with Period $k$}

Definition 1. Let $k$ be a positive integer. A function $f: \mathbb{R} \rightarrow$ $\mathbb{R}$ is said to be a Fibonacci function with period $k$ if $f(x+$ $2 k)=f(x+k)+f(x)$ for all $x \in \mathbb{R}$.
Example 2. Let $f(x)=a^{x / k}$ be a Fibonacci function with period $k \in \mathbb{N}$, where $a>0$. It follows that $a^{(x / k)+2}=a^{(x / k)+1}+$ $a^{x / k}$ for all $x \in \mathbb{R}$, so $a^{2}=a+1$. Then $a=(1+\sqrt{5}) / 2$. Thus, $f(x)=((1+\sqrt{5}) / 2)^{x / k}$ for all $x \in \mathbb{R}$.

Proposition 3. Let $f: \mathbb{R} \rightarrow \mathbb{R}$ be a Fibonacci function with period $k \in \mathbb{N}$. Assume that $f$ is differentiable. Then $f^{\prime}$ is also a Fibonacci function with period $k$.

Proof. Let $x \in \mathbb{R}$. Since $f(x+2 k)=f(x+k)+f(x)$, it follows that $f^{\prime}(x+2 k)=f^{\prime}(x+k)+f^{\prime}(x)$.

Proposition 4. Let $f: \mathbb{R} \rightarrow \mathbb{R}$ be a Fibonacci function with period $k \in \mathbb{N}$, and define $g_{t}(x)=f(x+t)$ for all $x \in \mathbb{R}$, where $t \in \mathbb{R}$. Then $g_{t}$ is also a Fibonacci function with period $k$.

Proof. Let $x \in \mathbb{R}$. Then $g_{t}(x+2 k)=f(x+2 k+t)=f(x+t+$ $k)+f(x+t)=g_{t}(x+k)+g_{t}(x)$.

Example 5. Let $k \in \mathbb{N}$ and $t \in \mathbb{R}$. Define $g_{t}: \mathbb{R} \rightarrow \mathbb{R}$ by $g_{t}(x)=((1+\sqrt{5}) / 2)^{(x+t) / k}$ for all $x \in \mathbb{R}$. Then $g_{t}$ is a Fibonacci function with period $k$.

Theorem 6. Let $f: \mathbb{R} \rightarrow \mathbb{R}$ be a Fibonacci function with period $k \in \mathbb{N}$, and let $\left\{F_{n}\right\}_{n \in \mathbb{N}}$ be a sequence of Fibonacci numbers with $F_{0}=0, F_{1}=1$, and $F_{n+1}=F_{n}+F_{n-1}$ for all $n \in \mathbb{N}$. Then, for any $n \in \mathbb{N}$ and $x \in \mathbb{R}, f(x+n k)=$ $F_{n} f(x+k)+F_{n-1} f(x)$. 
Proof. Let $x \in \mathbb{R}$. We note that $f(x+k)=F_{1} f(x+k)+F_{0} f(x)$ and $f(x+2 k)=F_{2} f(x+k)+F_{1} f(x)$. Now, we assume that $f(x+n k)=F_{n} f(x+k)+F_{n-1} f(x)$ and $f(x+(n+1) \mathrm{k})=$ $F_{n+1} f(x+k)+F_{n} f(x)$, where $n \in \mathbb{N}$. Then

$$
\begin{aligned}
f(x+ & (n+2) k) \\
& =f(x+(n+1) k)+f(x+n k) \\
& =F_{n+1} f(x+k)+F_{n} f(x)+F_{n} f(x+k)+F_{n-1} f(x) \\
& =\left(F_{n+1}+F_{n}\right) f(x+k)+\left(F_{n}+F_{n-1}\right) f(x) \\
& =F_{n+2} f(x+k)+F_{n+1} f(x) .
\end{aligned}
$$

This proof is completed.

\section{Odd Fibonacci Functions with Period $k$}

Definition 7. Let $k$ be a positive integer. A function $f: \mathbb{R} \rightarrow$ $\mathbb{R}$ is said to be an odd Fibonacci function with period $k$ if $f(x+2 k)=-f(x+k)+f(x)$ for all $x \in \mathbb{R}$.

Example 8. Let $f(x)=a^{x / k}$ be an odd Fibonacci function with period $k \in \mathbb{N}$, where $a>0$. It follows that $a^{(x / k)+2}=$ $-a^{(x / k)+1}+a^{x / k}$ for all $x \in \mathbb{R}$, so $a^{2}=-a+1$. Then $a=$ $(-1+\sqrt{5}) / 2$. Thus, $f(x)=((-1+\sqrt{5}) / 2)^{x / k}$ for all $x \in \mathbb{R}$.

Proposition 9. Let $f: \mathbb{R} \rightarrow \mathbb{R}$ be an odd Fibonacci function with period $k \in \mathbb{N}$. Assume that $f$ is differentiable. Then $f^{\prime}$ is also an odd Fibonacci function with period $k$.

Proof. Let $x \in \mathbb{R}$. Since $f(x+2 k)=-f(x+k)+f(x)$, it follows that $f^{\prime}(x+2 k)=-f^{\prime}(x+k)+f^{\prime}(x)$.

Proposition 10. Let $f: \mathbb{R} \rightarrow \mathbb{R}$ be an odd Fibonacci function with period $k \in \mathbb{N}$, and define $g_{t}(x)=f(x+t)$ for all $x \in \mathbb{R}$, where $t \in \mathbb{R}$. Then $g_{t}$ is also an odd Fibonacci function with period $k$.

Proof. Let $x \in \mathbb{R}$. Then $g_{t}(x+2 k)=f(x+2 k+t)=-f(x+$ $t+k)+f(x+t)=-g_{\mathrm{t}}(x+k)+g_{t}(x)$.

Example 11. Let $k \in \mathbb{N}$ and $t \in \mathbb{R}$. Define $g_{t}: \mathbb{R} \rightarrow \mathbb{R}$ by $g_{t}(x)=((-1+\sqrt{5}) / 2)^{(x+t) / k}$ for all $x \in \mathbb{R}$. Then $g_{t}$ is an odd Fibonacci function with period $k$.

Theorem 12. Let $f: \mathbb{R} \rightarrow \mathbb{R}$ be an odd Fibonacci function with period $k \in \mathbb{N}$, and let $\left\{F_{-n}\right\}_{n \in \mathbb{N}}$ be a sequence of Fibonucci numbers with $F_{0}=0, F_{-1}=1$, and $F_{-n-1}=-F_{-n}+F_{-n+1}$ for all $n \in \mathbb{N}$. Then, for any $n \in \mathbb{N}$ and $x \in \mathbb{R}, f(x+n k)=$ $F_{-n} f(x+k)+F_{-n+1} f(x)$.

Proof. Let $x \in \mathbb{R}$. We note that $f(x+k)=F_{-1} f(x+k)+F_{0} f(x)$ and $f(x+2 k)=F_{-2} f(x+k)+F_{-1} f(x)$. Now, we assume that $f(x+n k)=F_{-n} f(x+k)+F_{-n+1} f(x)$ and $f(x+(n+1) k)=$ $F_{-n-1} f(x+k)+F_{-n} f(x)$, where $n \in \mathbb{N}$. Then

$$
\begin{aligned}
f(x+ & (n+2) k) \\
= & -f(x+(n+1) k)+f(x+n k) \\
= & -\left(F_{-n-1} f(x+k)+F_{-n} f(x)\right) \\
& +F_{-n} f(x+k)+F_{-n+1} f(x) \\
= & \left(-F_{-n-1}+F_{-n}\right) f(x+k) \\
& +\left(-F_{-n}+F_{-n+1}\right) f(x) \\
= & F_{-n-2} f(x+k)+F_{-n-1} f(x) .
\end{aligned}
$$

This proof is completed.

\section{4. $f$-Even Functions with Period $k$}

Definition 13. Let $k$ be a positive integer and let $\alpha: \mathbb{R} \rightarrow \mathbb{R}$ be such that if $\alpha h=0$, where $h: \mathbb{R} \rightarrow \mathbb{R}$ is continuous, then $h=0$. The function $\alpha$ is said to be an $f$-even function with period $k$ if $\alpha(x+k)=\alpha(x)$ for all $x \in \mathbb{R}$.

Example 14. Define $\alpha(x)=x-\lfloor x\rfloor$ for all $x \in \mathbb{R}$. Let $h: \mathbb{R} \rightarrow$ $\mathbb{R}$ be a continuous function such that $\alpha h=0$. For any $x \notin \mathbb{Z}$, we have $\alpha(x) \neq 0$, so $h(x)=0$. Since $\mathbb{R} \backslash \mathbb{Z}$ is dense in $\mathbb{R}$ and $h$ is continuous, it follows that $h=0$. Let $k \in \mathbb{N}$ and $x \in \mathbb{R}$. Then $\alpha(x+k)=x+k-\lfloor x+k\rfloor=x+k-\lfloor x\rfloor-k=x-\lfloor x\rfloor=\alpha(x)$. Hence, $\alpha$ is an $f$-even function with period $k$.

Theorem 15. Let $k \in \mathbb{N}$ and $\alpha: \mathbb{R} \rightarrow \mathbb{R}$ be an $f$-even function with period $k$ and let $g: \mathbb{R} \rightarrow \mathbb{R}$ be a continuous function. Then $g$ is a Fibonacci function with period $k$ if and only if $\alpha g$ is a Fibonacci function with period $k$.

Proof. First, we assume that $g$ is a Fibonacci function with period $k$. For any $x \in \mathbb{R}$, we have

$$
\begin{aligned}
(\alpha g) & (x+2 k) \\
& =\alpha(x+2 k) g(x+2 k) \\
& =\alpha(x+k)(g(x+k)+g(x)) \\
& =\alpha(x+k) g(x+k)+\alpha(x+k) g(x) \\
& =\alpha(x+k) g(x+k)+\alpha(x) g(x) \\
& =(\alpha g)(x+k)+(\alpha g)(x) .
\end{aligned}
$$

Hence, $\alpha g$ is a Fibonacci function with period $k$.

Next, we assume that $\alpha g$ is a Fibonacci function with period $k$. Let $x \in \mathbb{R}$. Then

$$
\begin{aligned}
\alpha(x+ & k) g(x+2 k) \\
& =\alpha(x+2 k) g(x+2 k) \\
& =(\alpha g)(x+2 k) \\
& =(\alpha g)(x+k)+(\alpha g)(x)
\end{aligned}
$$




$$
\begin{aligned}
& =\alpha(x+k) g(x+k)+\alpha(x) g(x) \\
& =\alpha(x+k) g(x+k)+\alpha(x+k) g(x) \\
& =\alpha(x+k)(g(x+k)+g(x)) .
\end{aligned}
$$

By the assumption of $\alpha$, we obtain that $g(x+2 k)=g(x+$ $k)+g(x)$. Hence, $g$ is a Fibonacci function with period $k$.

Example 16. Let $k \in \mathbb{N}$. Define $\alpha(x)=x-\lfloor x\rfloor$ and $g(x)=$ $((1+\sqrt{5}) / 2)^{x / k}$ for all $x \in \mathbb{R}$. For all $x \in \mathbb{R}$, we have $\alpha g(x)=(x-\lfloor x\rfloor)((1+\sqrt{5}) / 2)^{x / k}$. We recall that $\alpha$ is an $f$ even function with period $k$, and $g$ is a Fibonacci function with period $k$. Hence, $\alpha g$ is a Fibonacci function with period $k$.

Theorem 17. Let $k \in \mathbb{N}$ and $\alpha: \mathbb{R} \rightarrow \mathbb{R}$ be an $f$-even function with period $k$ and let $g: \mathbb{R} \rightarrow \mathbb{R}$ be a continuous function. Then $g$ is an odd Fibonacci function with period $k$ if and only if $\alpha g$ is an odd Fibonacci function with period $k$.

Proof. First, we assume that $g$ is an odd Fibonacci function with period $k$. For any $x \in \mathbb{R}$, we have

$$
\begin{aligned}
(\alpha g) & (x+2 k) \\
& =\alpha(x+2 k) g(x+2 k) \\
& =\alpha(x+k)(-g(x+k)+g(x)) \\
& =-\alpha(x+k) g(x+k)+\alpha(x+k) g(x) \\
& =-\alpha(x+k) g(x+k)+\alpha(x) g(x) \\
& =-(\alpha g)(x+k)+(\alpha g)(x) .
\end{aligned}
$$

Hence, $\alpha g$ is an odd Fibonacci function with period $k$.

Next, we assume that $\alpha g$ is an odd Fibonacci function with period $k$. Let $x \in \mathbb{R}$. Then

$$
\begin{aligned}
\alpha(x+ & k) g(x+2 k) \\
& =\alpha(x+2 k) g(x+2 k) \\
& =(\alpha g)(x+2 k) \\
& =-(\alpha g)(x+k)+(\alpha g)(x) \\
& =-\alpha(x+k) g(x+k)+\alpha(x) g(x) \\
& =-\alpha(x+k) g(x+k)+\alpha(x+k) g(x) \\
& =\alpha(x+k)(-g(x+k)+g(x)) .
\end{aligned}
$$

By the assumption of $\alpha$, we obtain that $g(x+2 k)=-g(x+$ $k)+g(x)$. Hence, $g$ is an odd Fibonacci function with period $k$.

Example 18. Let $k \in \mathbb{N}$. Define $\alpha(x)=x-\lfloor x\rfloor$ and $g(x)=$ $((-1+\sqrt{5}) / 2)^{x / k}$ for all $x \in \mathbb{R}$. For all $x \in \mathbb{R}$, we have $\alpha g(x)=$ $(x-\lfloor x\rfloor)((-1+\sqrt{5}) / 2)^{x / k}$. We recall that $\alpha$ is an $f$-even function with period $k$ and $g$ is an odd Fibonacci function with period $k$. Hence, $\alpha g$ is an odd Fibonacci function with period $k$.

\section{5. $f$-Odd Functions with Period $k$}

Definition 19. Let $k$ be a positive integer and let $\alpha: \mathbb{R} \rightarrow \mathbb{R}$ be such that if $\alpha h=0$ where $h: \mathbb{R} \rightarrow \mathbb{R}$ is continuous, then $h=0$. The function $\alpha$ is said to be an $f$-odd function with period $k$ if $\alpha(x+k)=-\alpha(x)$ for all $x \in \mathbb{R}$.

Example 20. Define $\alpha(x)=\sin (\pi x)$ for all $x \in \mathbb{R}$. Let $h$ : $\mathbb{R} \rightarrow \mathbb{R}$ be a continuous function such that $\alpha h=0$. For any $x \notin \pi \mathbb{Z}$, we have $\alpha(x) \neq 0$, so $h(x)=0$. Since $\mathbb{R} \backslash \pi \mathbb{Z}$ is dense in $\mathbb{R}$ and $h$ is continuous, it follows that $h=0$. Let $k$ be a positive odd integer and $x \in \mathbb{R}$. Then $\alpha(x+k)=\sin (\pi(x+k))=$ $\sin (\pi x+\pi k)=-\sin (\pi x)=-\alpha(x)$. Hence, $\alpha$ is an $f$-even function with period $k$.

Theorem 21. Let $k \in \mathbb{N}$ and $\alpha: \mathbb{R} \rightarrow \mathbb{R}$ be an $f$-odd function with period $k$ and let $g: \mathbb{R} \rightarrow \mathbb{R}$ be a continuous function. Then $g$ is a Fibonacci function with period $k$ if and only if $\alpha g$ is an odd Fibonacci function with period $k$.

Proof. First, we assume that $g$ is a Fibonacci function with period $k$. For any $x \in \mathbb{R}$, we have

$$
\begin{aligned}
(\alpha g) & (x+2 k) \\
& =\alpha(x+2 k) g(x+2 k) \\
& =-\alpha(x+k)(g(x+k)+g(x)) \\
& =-\alpha(x+k) g(x+k)-\alpha(x+k) g(x) \\
& =-\alpha(x+k) g(x+k)+\alpha(x) g(x) \\
& =-(\alpha g)(x+k)+(\alpha g)(x) .
\end{aligned}
$$

Hence, $\alpha g$ is an odd Fibonacci function with period $k$.

Next, we assume that $\alpha g$ is an odd Fibonacci function with period $k$. Let $x \in \mathbb{R}$. Then

$$
\begin{aligned}
\alpha(x+ & k) g(x+2 k) \\
& =-\alpha(x+2 k) g(x+2 k) \\
& =-(\alpha g)(x+2 k) \\
& =-(-(\alpha g)(x+k)+(\alpha g)(x)) \\
& =(\alpha g)(x+k)-(\alpha g)(x) \\
& =\alpha(x+k) g(x+k)-\alpha(x) g(x) \\
& =\alpha(x+k) g(x+k)+\alpha(x+k) g(x) \\
& =\alpha(x+k)(g(x+k)+g(x)) .
\end{aligned}
$$

By the assumption of $\alpha$, we obtain that $g(x+2 k)=g(x+$ $k)+g(x)$. Hence, $g$ is a Fibonacci function with period $k$. 
Example 22. Let $k$ be a positive odd integer. Define $\alpha(x)=$ $\sin (\pi x)$ and $g(x)=((1+\sqrt{5}) / 2)^{x / k}$ for all $x \in \mathbb{R}$. We have $\alpha g(x)=(\sin (\pi x))((1+\sqrt{5}) / 2)^{x / k}$ for all $x \in \mathbb{R}$. We recall that $\alpha$ is an $f$-odd function with period $k$ and $g$ is a Fibonacci function with period $k$. Hence, $\alpha g$ is an odd Fibonacci function with period $k$.

Theorem 23. Let $k \in \mathbb{N}$ and $\alpha: \mathbb{R} \rightarrow \mathbb{R}$ be an $f$-odd function with period $k$ and let $g: \mathbb{R} \rightarrow \mathbb{R}$ be a continuous function. Then $g$ is an odd Fibonacci function with period $k$ if and only if $\alpha g$ is a Fibonacci function with period $k$.

Proof. First, we assume that $g$ is an odd Fibonacci function with period $k$. For any $x \in \mathbb{R}$, we have

$$
\begin{aligned}
(\alpha g) & (x+2 k) \\
& =\alpha(x+2 k) g(x+2 k) \\
& =-\alpha(x+k)(-g(x+k)+g(x)) \\
& =\alpha(x+k) g(x+k)-\alpha(x+k) g(x) \\
& =\alpha(x+k) g(x+k)+\alpha(x) g(x) \\
& =(\alpha g)(x+k)+(\alpha g)(x) .
\end{aligned}
$$

Hence, $\alpha g$ is a Fibonacci function with period $k$.

Next, we assume that $\alpha g$ is a Fibonacci function with period $k$. Let $x \in \mathbb{R}$. Then

$$
\begin{aligned}
\alpha(x+ & k) g(x+2 k) \\
& =-\alpha(x+2 k) g(x+2 k) \\
& =-(\alpha g)(x+2 k) \\
& =-((\alpha g)(x+k)+(\alpha g)(x)) \\
& =-(\alpha g)(x+k)-(\alpha g)(x) \\
& =-\alpha(x+k) g(x+k)-\alpha(x) g(x) \\
& =-\alpha(x+k) g(x+k)+\alpha(x+k) g(x) \\
& =\alpha(x+k)(-g(x+k)+g(x)) .
\end{aligned}
$$

By the assumption of $\alpha$, we obtain that $g(x+2 k)=-g(x+$ $k)+g(x)$. Hence, $g$ is an odd Fibonacci function with period $k$.

Example 24. Let $k$ be a positive odd integer. Define $\alpha(x)=$ $\sin (\pi x)$ and $g(x)=((-1+\sqrt{5}) / 2)^{x / k}$ for all $x \in \mathbb{R}$. We have $\alpha g(x)=(\sin (\pi x))((-1+\sqrt{5}) / 2)^{x / k}$ for all $x \in \mathbb{R}$. We recall that $\alpha$ is an $f$-odd function with period $k$ and $g$ is an odd Fibonacci function with period $k$. Hence, $\alpha g$ is a Fibonacci function with period $k$.

\section{Open Problems}

Conjecture 25. If $f$ is a Fibonaccifunction with period $k \in \mathbb{N}$, then

$$
\lim _{x \rightarrow \infty} \frac{f(x+k)}{f(x)}=\frac{1+\sqrt{5}}{2} .
$$

Conjecture 26. If $f$ is an odd Fibonacci function with period $k \in \mathbb{N}$, then

$$
\lim _{x \rightarrow \infty} \frac{f(x+k)}{f(x)}=\frac{-1-\sqrt{5}}{2} .
$$

\section{Acknowledgments}

The author would like to thank the referees for their useful comments and suggestions.

\section{References}

[1] K. T. Atanassov, V. Atanassova, A. G. Shannon, and J. C. Turner, New Visual Perspectives on Fibonacci Numbers, World Scientific Publishing, Hackensack, NJ, USA, 2002.

[2] R. A. Dunlap, The Golden Ratio and Fibonacci Numbers, World Scientific Publishing, Hackensack, NJ, USA, 1997.

[3] H. S. Kim and J. Neggers, "Fibonacci mean and golden section mean," Computers \& Mathematics with Applications, vol. 56, no. 1, pp. 228-232, 2008.

[4] S.-M. Jung, "Hyers-Ulam stability of Fibonacci functional equation," Iranian Mathematical Society. Bulletin, vol. 35, no. 2, pp. 217-227, 2009.

[5] J. S. Han, H. S. Kim, and J. Neggers, "The Fibonacci-norm of a positive integer: observations and conjectures," International Journal of Number Theory, vol. 6, no. 2, pp. 371-385, 2010.

[6] J. S. Han, H. S. Kim, and J. Neggers, "Fibonacci sequences in groupoids," Advances in Difference Equations, vol. 2012, article 19, 2012.

[7] J. S. Han, H. S. Kim, and J. Neggers, "On Fibonacci functions with Fibonacci numbers," Advances in Difference Equations, vol. 2012, article 126, 2012. 


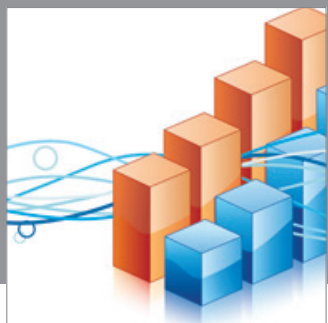

Advances in

Operations Research

mansans

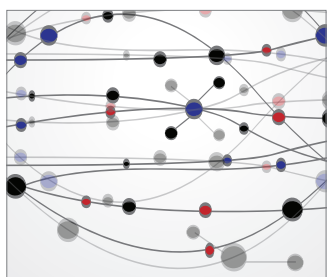

The Scientific World Journal
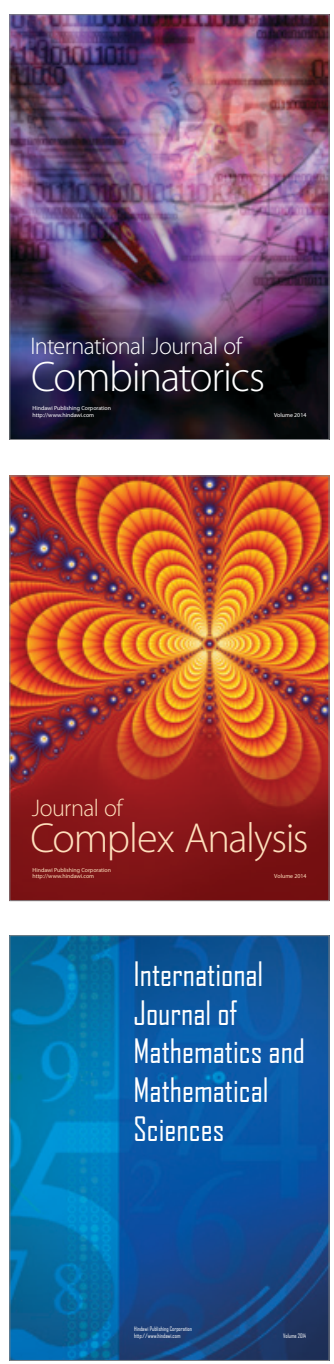
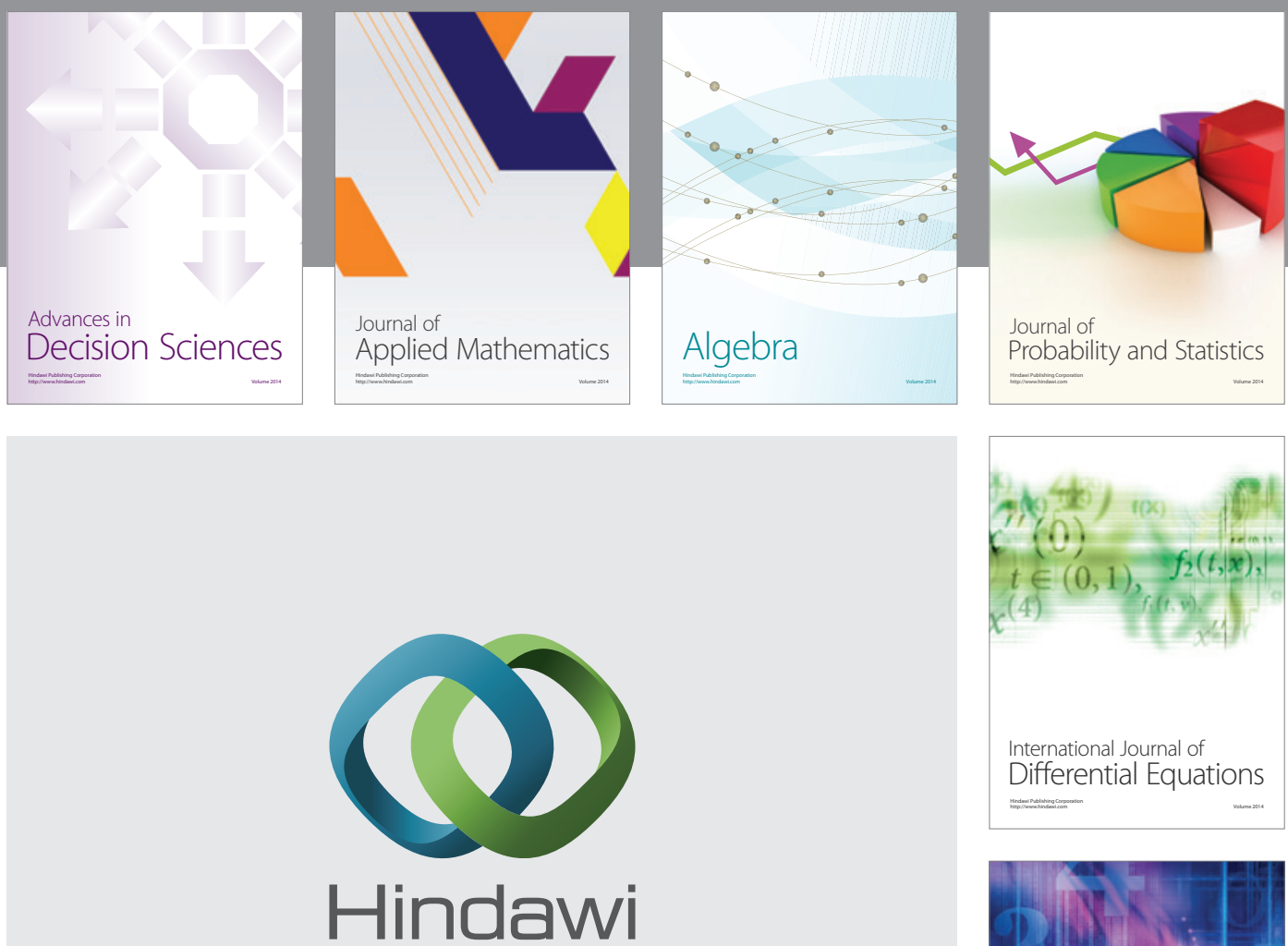

Submit your manuscripts at http://www.hindawi.com
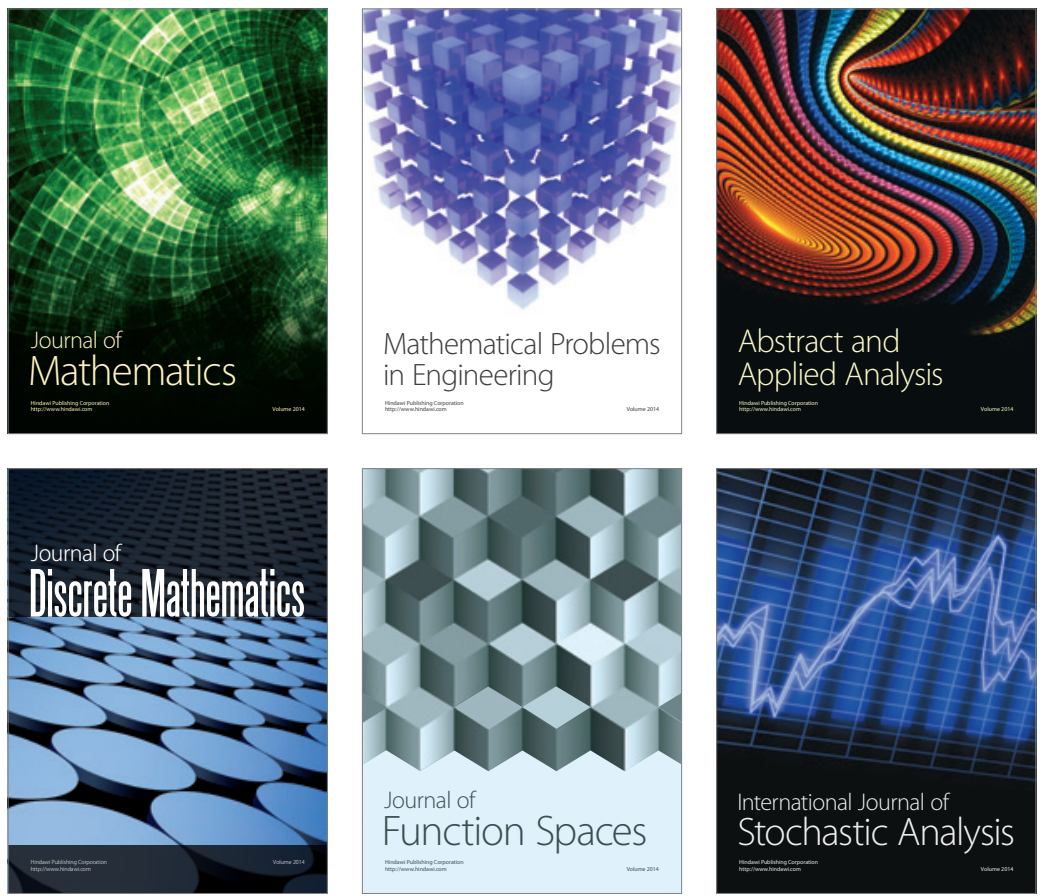

Journal of

Function Spaces

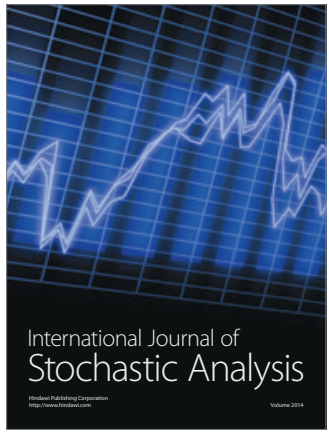

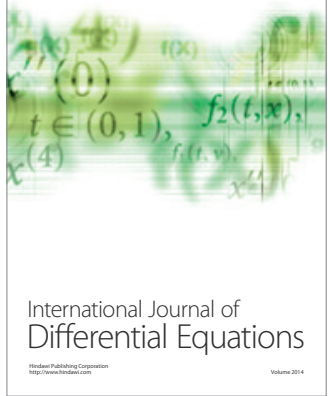
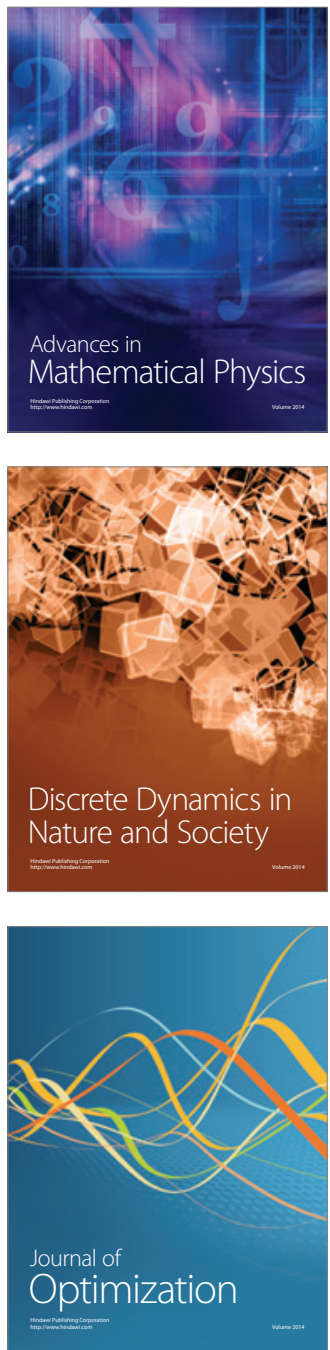